\title{
FESTSCHRIFT FOR EROL GELENBE'S 70TH BIRTHDAY
}

\author{
Nihal Pekergin \\ Laboratoire $L A C L$ \\ Université Paris-Est Créteil Val-de-Marne \\ 61 av. du Général de Gaulle \\ 94010 Créteil Cedex \\ France \\ E-mail: nihal.pekergin@u-pec.fr
}

\begin{abstract}
This paper introduces a special collection of research contributions written to honour Professor Erol Gelenbe of his 70th birthday which was celebrated on September 20-25, 2015 at Imperial College in London where he holds the Dennis Gabor Professorship and is Head of the Intelligent Systems and Networks Group in the Department of Electrical and Electronic Engineering (see http://san.ee.ic.ac.uk/Gelenbe2015). All but three of the fourteen papers presented here were written by his students and descendants. These papers are centered on probability models related to computer systems and networks, an area where Erol plays a pioneering role in which he remains very active with many innovations and new directions that he has introduced in the last ten or twelve years. We first briefly review Erol's work in these areas, and then discuss each of the contributions that appear here together with their links to Erol's own published research.
\end{abstract}

\section{THIS SPECIAL ISSUE}

This special issue is a collection of fourteen papers that honor Erol Gelenbe, on the occasion of his 70th birthday (Figures 1 and 2). Most of them were presented at the Symposium that was held to celebrate his work from September 20-25th at Imperial College and other venues in London.

Erol's work in probability modelling of computations and computer systems and networks started with his Ph.D. Thesis at the Polytechnic Institute of New York in Brooklyn, better known as Brooklyn Poly, under the supervision of Professor Edward J. Smith Jr. Erol's productive work as a researcher, Ph.D. supervisor, industrial consultant and technical innovator has continued throughout his career at the University of Michigan, Ann Arbor, and then at the University of Liège, at INRIA, at the Universities of Paris-Nord, Paris-Sud (Orsay), Paris V (René Descartes) where he founded its Computer Science Department (Ecole des Hautes Etudes en Informatique), at the New Jersey Institute of Technology 


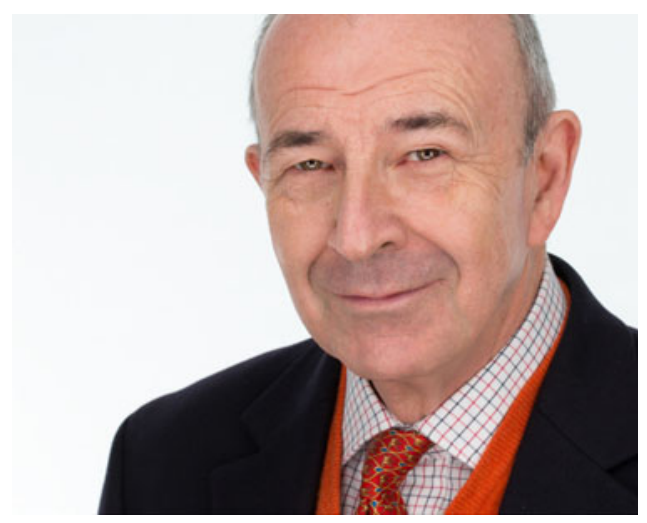

Figure 1. Portrait of Professor Erol Gelenbe, photographed on 14th January 2016 by Dr. Peter Weiner in Paris. Dr. Weiner was the founding Chair of the Computer Science Department of Yale University and former faculty member at Princeton, then researcher at the Rand Corporation. A man of many talents, Dr. Weiner studied (like Erol) under Professor Edward J. Smith at the Polytechnic Institute of Brooklyn in New York City. Dr. Weiner then went on to become a successful entrepreneur, as well as a lawyer and photographer.

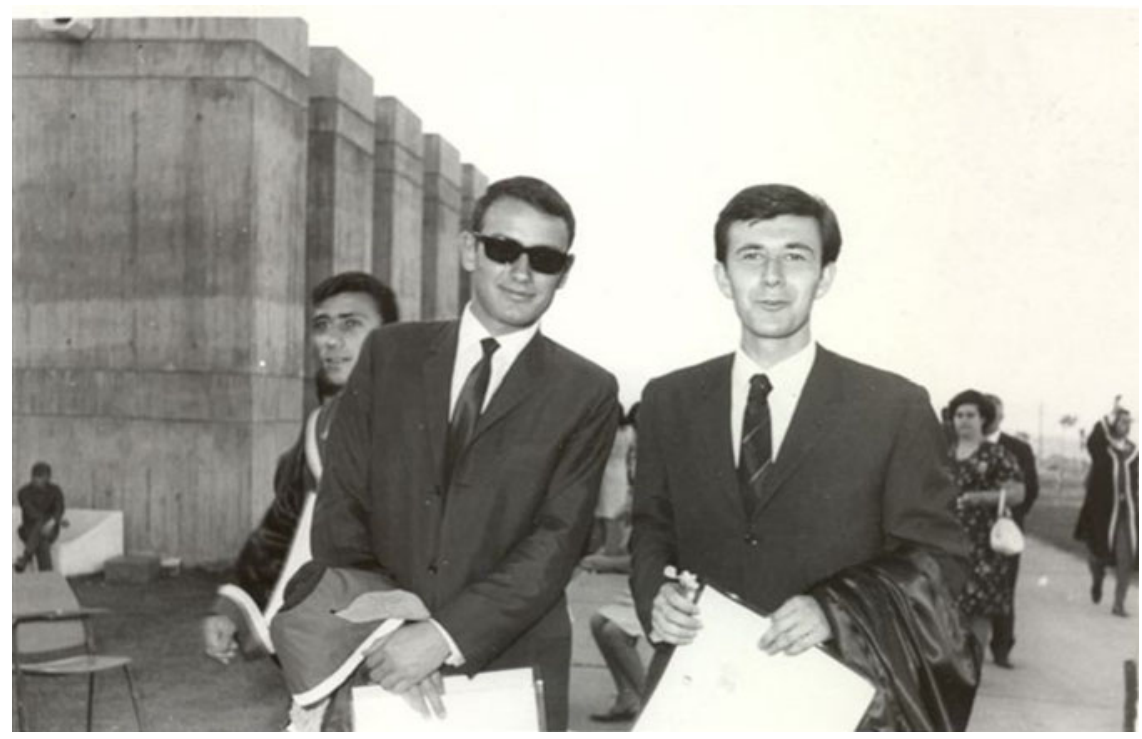

Figure 2. Erol Gelenbe (right) on his graduation day from the Middle East Technical University with a Bachelor of Science degree (High Honours) in June 1966, in Ankara (Turkey). On the left side is his good friend and high-school class mate (from Ankara Koleji) Polat Gülkan, who was graduating from Civil Engineering. Professor Gülkan went on to become a distinguished seismologist and an expert on the construction of buildings that are resistant to earthquakes. 
where he briefly held the New Jersey State Endowed Chair of Computer Science, at Duke University, the University of Central Florida, and now at Imperial College since 2003.

His earliest work studied the deterministic design of checking experiments for finitestate machines [9] and the realization of finite state machines from many identical small modules [47].

Then he turned to the mathematical properties of finite state automata with probabilistic transitions [13], and the types of formal languages that they would recognize $[11,12]$, showing that not only the probabilistic transitions themselves but also the probability that is used as a threshold for word recognition can have a powerful effect on the family of formal languages that are recognized.

Under the influence of the hot topic of the day, which was the design of virtual memory computer time-sharing systems, after joining the University of Michigan, Ann Arbor, and influenced by the design needs of the Michigan Time Sharing System, he turned his attention to the optimization of memory management systems [14-16,52], the overall realtime management of time-sharing systems [46] as well as the analysis of page replacement algorithms [17].

Driven by the need to understand how the performance of computation can be characterized in a general way, to include not just the counting of computational steps or of memory units (as is usually done in complexity theory) but also the interference between different programs and jobs executing on the same machine, the effect of the memory hierarchy and architecture, the time it takes to transfer data in and out of secondary memory, the processor to memory interconnections, the CPU scheduling algorithms, the effect of the user terminals and other input-output devices, and the interactions between all these aspects including the operating systems, he continued over the years with a unified variety of topics focusing on system performance, including energy consumption and security in the most recent years.

This work has included the performance of parallel numerical algorithms [39], the intrinsic mathematical properties of secondary memory systems and their latency [36], the uncertainty related to information itself [33], the end-to-end delay of important protocols such as Voice-over-IP that is now many years later used in Skype [3], the performance of search algorithms in unknown and dangerous environments [29], the behavior of interacting real and virtual agents within a simulation [35], the performance of gene regulatory networks [22], adaptive schemes in packet routing [21], the energy consumed in packet networks [43], or in communicating with spins $[27,28]$.

Several papers in this special issue are directly related to various epochs of Erol's research. Thus, the first paper by Ufuk Cag̃layan focuses its review mostly on Erol's relatively recent work over the last 10 to 15 years.

The second paper by Guy Fayolle and Paul Muhlethaler on "A Markovian Analysis of IEEE 802.11 Broadcast Transmission Networks with Buffering proposes two models for a Carrier Sense Multiple Access (CSMA) protocol close to IEEE 802.11. This links directly to some of Erol's and Guy Fayolle's early work from the 1970s, and to Erol's early work on CSMA $[6,7,42]$. The authors of this paper compute the station transmission rate, the stability conditions, and the average packet delay for protocols that yield stable behavior without external control, in contrast to systems such as ALOHA and CSMA. The authors' numerical results also match well the performance of the standard IEEE 802.11 broadcast protocol.

The paper by Andrea Marin on "Product-form in G-networks" reviews the well known, and less known, results on Erol's famous Gelenbe or G-Networks [20,38]. Erol invented these models to characterize systems where customers or users have the ability to influence the behavior of others, such as re-routing them towards different resources than the ones 
they have originally requested, or removing them altogether from the system either singly or in batches. These behaviors result in unusual non-linear traffic equations, and Erol had shown that they he have a separable and computationally efficient product form solution [45]. Dr. Marin's paper discusses the equational characteristics which lead to the product form.

Similarly, the paper by Jean-Michel Fourneau on "G-networks of unreliable nodes shows an interesting generalization where individual nodes may be subject to breakdowns, but the important aspect is that they are provoked by arrivals of some specific customers which are the breakdowns. The paper introduces this model and establishes the product form solution.

Stelios Timotheou in his paper "Fast Non-negative Least Squares Learning in the Random Neural Network (RNN)" discusses a fast learning algorithm for the RNN [31], introduced in the late 1980's and the 1990's [19,32,49,50,53]. It has had applications in image processing $[30,37,51]$ and network routing [24]. The model's capability to approximate arbitrary continuous and bounded functions [41] is the theoretical justification for the use of learning algorithms that store complex functional relationships within these models.

The next paper on "On-Road Vehicle Classification Based on Random Neural Network and Bag-of-Visual Words" by Khaled F. Hussain and Ghada S. Moussa is a continuation of work conducted several years ago by Dr Hussain with Erol in two directions: learning algorithms for stochastic neural networks [34], and their use in the important practical problem of the recognition of the class to which a given vehicle belongs (e.g. cars, trucks, motorcycles, etc.) from imaging [1].

On the other hand, the work by Tug̃rul Dayar and M. Can Orhan on "Cartesian product partitioning of multi-dimensional reachable state spaces" studies Markov chains that are composed of interacting subsystems, so that they can be constructed from a multidimensional model with each subsystem corresponding to a different dimension. This topic is related to Erol's Ph.D. work from the 1960s and 1970s [10,47] on the study of state-space partitions for stochastic and deterministic system decomposition. The authors establish that the partitioning of the reachable state space of a three or higher dimensional system with a minimum number of partitions into Cartesian products of subsets of state spaces is NP-complete, and discuss both a merge based and a refinement-based algorithm that yield possibly non-optimal partitions, and then they provide some experiments using these algorithms.

When dealing with multiple agents that have to take local decisions cooperatively based on limited interaction with their peers, the issue of determining whether they behave in an overall optimal fashion is an old and well known problem that is encountered in many diverse and unusual applications such as the control of multiple updates to a distributed database [48], the access of several processes to common data [4], defense against Distributed Denial of Service attacks [40], search by multiple agents [23,26], auctions of different buyers within a shared economy [25], or emergency management where many evacuees have to exit a common area $[5,8,54]$. In the paper by Bruno Gaujal and Panayotis Mertikopoulos, "A Distributed Stochastic Approximation Algorithm for Stochastic Semi-definite Programming", a distributed stochastic approximation algorithm is presented, motivated by communications between multiple agents using common resources, such as shared wireless environments with Multiple-Input-Multiple-Output. The authors establish that their approach converges to the optimum under fairly general conditions and illustrate this with numerical examples.

The next paper is the work of Olivier Brun on "Performance of non-cooperative routing over parallel non-observable queues" in which recent results on non-cooperative loadbalancing games for autonomic computing are presented. The following paper by Yingdong Lu, Mayank Sharma, Mark S Squillante and Bo Zhang on "Stochastic Optimal Dynamic Control of $G I_{m} / G I_{m} / 1_{n}$ Queues with Time-Varying Workloads" addresses one of the many 
issues that are coming to the forefront of the control of computer systems, including energy issues [43].

Then, Efrat Perel and Uri Yechiali in their paper "Finite Two Layered Queueing Systems" study matrix geometric analytical techniques for large state-space systems arising in queueing theory.

The 12th paper is authored by Omer H. Abdelrahman and discusses "Detecting Network-Unfriendly Mobiles With the Random Neural Network". It links two areas in which Erol Gelenbe is active: network anomaly and attack detection techniques [55] and RNNs [18]. It exploits the RNN's capability to learn from examples so as to classify the attack patterns that are observed in mobile networks, so as to detect such attacks.

The final paper by Huibo Bi and Omer H. Abdelrahman, discusses energy aware navigation of vehicles. It develops an energy optimization framework akin to the work in $[43,44]$, which also includes specific energy consumption data related to motor vehicles. Then the paper applies this approach to a specific instance and evaluates it by simulations using the building evacuation simulator DBES $[2,5,54,56]$.

Thus, this rich and diverse set of 14 papers from France (5), the USA (1), Turkey (2), Italy (1), Cyprus (1), Israel (1), Egypt (1), and the UK (2), most of which are in direct relation to his own work, is a fitting tribute indeed to Erol's many research contributions to developing applied probability models in the context of the engineering and information sciences, and to the leadership that he exerts for many decades on this field.

\section{ABOUT THE AUTHOR}

Nihal Pekergin was born in Sivas, Turkey, and received her Engineering Degree in Electronics and Telecommunications from the Istanbul Technical University in 1983. She received a Master of Science degree in Computer Science from the same university in 1986. In 1987, she was awarded a Research Master's degree (DEA) in Modeling of Computer Systems from the Université Paris-Sud in 1987, and in 1991 she was awarded a Ph.D. on "New performance models of parallel systems" under the supervision of Erol Gelenbe at the Université de Paris V.

Professor Pekergin started her academic career at the Istanbul Technical University where she served as an Assistant from 1986 to 1986. From 1988 to 1989 she was a Visiting Assistant at the Université de Paris-Sud, and then a Temporary Lecturer at the same university till 1991. In 1991, she was appointed to a Maître de Conférences position at the Sorbonne (Université de Paris I) where she remained until 2007.

In the meanwhile, she passed her Habilitation degree in 2001 at the University of Versailles-St-Quentin on "Performance Bounds for Networks and Systems", and joined the Université Paris-Est Créteil Val de Marne in September 2007. There, she served as Head of the Computer Science Department in the Faculty of Sciences and Technology (2008-2014). She has published several refereed international conference and journal papers, and is active in the formal aspects of the field of performance evaluation and quantitative verification.

\section{References}

1. Abdelbaki, H.M., Hussain, K., \& Gelenbe, E. (2001). A laser intensity image based automatic vehicle classification system. In 2001 IEEE Proceedings on Intelligent Transportation Systems, 2001, Oakland, USA, August 2001. IEEE, pp. 460-465.

2. Akinwande, O.J., Bi, H., \& Gelenbe, E. (2015). Managing crowds in hazards with dynamic grouping. IEEE Access 3: 1060-1070. [Online]. Available: http://dx.doi.org/10.1109/ACCESS.2015.2453341

3. Baccelli, F., Gelenbe, E., \& Plateau, B. (1984). An end-to-end approach to the resequencing problem. Journal of the ACM 31(3): 474-485. [Online]. Available: http://doi.acm.org/10.1145/828.1883 
4. Chesnais, A., Gelenbe, E., \& Mitrani, I. (1983). On the modeling of parallel access to shared data. Communications of the ACM 26(3): 196-202. [Online]. Available: http://doi.acm.org/10.1145/358061. 358073

5. Dimakis, N., Filippoupolitis, A., \& Gelenbe, E. (2010). Distributed building evacuation simulator for smart emergency management. Computer Journal 53(9): 1384-1400. [Online]. Available: http://dx.doi.org/10.1093/comjnl/bxq012

6. Fayolle, G., Gelenbe, E., Labetoulle, J., \& Bastin, D. (1974). The stability problem of broadcast packet switching computer networks. Acta Informatica 4(1): 49-53.

7. Fayolle, G., Gelenbe, E., \& Labetoulle, J. (1977). Stability and optimal control of the packet switching broadcast channel. Journal of the ACM 24(3): 375-386.

8. Filippoupolitis, A., Hey, L., Loukas, G., Gelenbe, E., \& Timotheou, S. (2008). Emergency response simulation using wireless sensor networks. In Proceedings of the First International Conference on Ambient Media and Systems, Quebec, Canada, February 2008. ICST (Institute for Computer Sciences, Social-Informatics and Telecommunications Engineering), p. 21.

9. Gelenbe, S.E. (1967). Regular expressions and checking experiments. Polytechnic Institute of Brooklyn N.Y.: Microwave Research Institute, September.

10. Gelenbe, S.E. (1969). On probabilistic automata with structural restrictions. In IEEE Conference Record of Tenth Annual Symposium on Switching and Automata Theory, Waterloo, Canada, November 1969.

11. Gelenbe, E. (1970). On languages defined by linear probabilistic automata. Information and Control 16(5): 487-501.

12. Gelenbe, E. (1970). On the loop-free decomposition of stochastic finite-state systems. Information and Control 17(5): 474-484.

13. Gelenbe, S.E. (1971). A realizable model for stochastic sequential machines. IEEE Transactions on Computers C-20(2): 199-204.

14. Gelenbe, E. (1971). The two-thirds rule for dynamic storage allocation under equilibrium. Information Processing Letters 1(2): 59-60.

15. Gelenbe, E. (1973). The distribution of a program in primary and fast buffer storage. Commununications of the ACM 16(7): 431-434.

16. Gelenbe, E. (1973). Minimizing wasted space in partitioned segmentation. Communnunications of the ACM 16(6): 343-349.

17. Gelenbe, E. (1973). A unified approach to the evaluation of a class of replacement algorithms. IEEE Transactions on Computers 22(6): 611-618.

18. Gelenbe, E. (1990). Stability of the random neural network model. Neural Computation 2(2): 239-247.

19. Gelenbe, E. (1998). The spiked random neural network: nonlinearity, learning and approximation. In Proceedings of 1998 Fifth IEEE International Workshop on Cellular Neural Networks and Their Applications, London, UK, April 1998. IEEE, pp. 14-19.

20. Gelenbe, E. (2000). The first decade of g-networks. European Journal of Operational Research 126(2): 231-232.

21. Gelenbe, E. (2003). Sensible decisions based on QOS. Computational Management Science 1(1): 1-14.

22. Gelenbe, E. (2007). Steady-state solution of probabilistic gene regulatory networks. Physical Review E 76(3): 031903.

23. Gelenbe, E. (2007). A diffusion model for packet travel time in a random multihop medium. $A C M$ Transactions on Sensor Networks 3(2): 10.

24. Gelenbe, E. (2009). Steps toward self-aware networks. Communications of the ACM 52(7): 66-75.

25. Gelenbe, E. (2009). Analysis of single and networked auctions. ACM Transactions on Internet Technology 9(2): 8 .

26. Gelenbe, E. (2010). Search in unknown random environments. Physical Review E 82(6): 061112.

27. Gelenbe, E. (2014). Error and energy when communicating with spins. In 2014 IEEE Global Conference on Signal and Information Processing (GlobalSIP), Atlanta, USA, December 2014. IEEE, pp. 784-787.

28. Gelenbe, E. (2015). Errors and power when communicating with spins. IEEE Transactions on Emerging Topics Computing 3(4): 483-488. [Online]. Available: http://doi.ieeecomputersociety.org/10.1109/ TETC.2015.2446751

29. Gelenbe, E. \& Cao, Y. (1998). Autonomous search for mines. European Journal of Operational Research 108(2): 319-333.

30. Gelenbe, E., Feng, Y., Ranga, K., \& Krishnan, R. (1996). Neural networks for volumetric mr imaging of the brain. In Proceedings of International Workshop on Neural Networks for Identification, Control, Robotics, and Signal/Image Processing, 1996, Venice, Italy, August 1996. IEEE, pp. 194-202.

31. Gelenbe, E. \& Fourneau, J.-M. (1999). Random neural networks with multiple classes of signals. Neural Computation 11(4): 953-963. 
32. Gelenbe, E., Harmanı, K., \& Krolik, J. (1998). Learning neural networks for detection and classification of synchronous recurrent transient signals. Signal Processing 64(3): 233-247.

33. Gelenbe, E. \& Hebrail, G. (1986). A probability model of uncertainty in data bases. In Proceedings of the Second International Conference on Data Engineering, Los Angeles, USA, February 1986. IEEE Computer Society, pp. 328-333.

34. Gelenbe, E. \& Hussain, K. (2002). Learning in the multiple class random neural network. IEEE Transactions on Neural Networks 13(6): 1257-1267. [Online]. Available: http://dx.doi.org/10.1109/TNN.2002. 804228

35. Gelenbe, E., Hussain, K., \& Kaptan, V. (2005). Simulating autonomous agents in augmented reality. Journal of Systems and Software 74(3): 255-268.

36. Gelenbe, E. \& Iasnogorodski, R. (1980). A queue with server of walking type (autonomous service). Annales de l'Institut Henri Poincaré. Section B, Calcul des Probabilités et Statistique 16(1): 63-73.

37. Gelenbe, E. \& Koçak, T. (2000). Area-based results for mine detection. IEEE Transactions on Geoscience and Remote Sensing 38(1): 12-24.

38. Gelenbe, E. \& Labed, A. (1998). G-networks with multiple classes of signals and positive customers. European Journal of Operational Research 108(2): 293-305.

39. Gelenbe, E., Lichnewsky, A., \& Stafylopatis, A. (1982). Experience with the parallel solutions of partial differential equations on a distributed computing system. IEEE Transactions on Computers 31(12): 1157-1164. [Online]. Available: http://doi.ieeecomputersociety.org/10.1109/TC.1982.1675939

40. Gelenbe, E. \& Loukas, G. (2007). A self-aware approach to denial of service defence. Computer Networks 51(5): 1299-1314.

41. Gelenbe, E., Mao, Z.-W., \& Li, Y.-D. (1999). Function approximation with spiked random networks. IEEE Transactions on Neural Networks 10(1): 3-9.

42. Gelenbe, E. \& Mitrani, I. (1982). Control policies in CSMA local area networks: ethernet controls. $A C M$ SIGMETRICS Performance Evaluation Review, ACM 11(4): 233-240.

43. Gelenbe E. \& Morfopoulou, C. (2011). A framework for energy-aware routing in packet networks. The Computer Journal 54(6): 850-859.

44. Gelenbe, E. \& Morfopoulou, C. (2011). Routing and g-networks to optimise energy and quality of service in packet networks. In Energy-Efficient Computing and Networking. Berlin, Heidelberg: Springer, pp. $163-173$.

45. Gelenbe, E. \& Muntz, R.R. (1976). Probabilistic models of computer systems: Part I (exact results). Acta Informatica 7(1): 35-60.

46. Gelenbe, E., Potier, D., Brandwajn, A., \& Lenfant, J. (1973). Gestion optimale d'un ordinateur multiprogramme à mémoire virtuelle. In Fifth Conference on Optimization Techniques, Part 2, Rome, Italy, May 7-11, 1973, ser. Lecture Notes in Computer Science, eds R. Conti \& A. Ruberti. vol. 4. Berlin, Heidelberg: Springer, pp. 132-143.

47. Gelenbe, E. \& Rossi, N. (1971). Uniform modular realizations and linear machines. IEEE Transactions on Computers 20(12): 1616-1617.

48. Gelenbe, E. \& Sevcik, K. (1979). Analysis of update synchronization for multiple copy data bases. IEEE Transactions on Computers 28(10): 737-747.

49. Gelenbe, E. \& Stafylopatis, A. (1991). Global behaviour of homogenous random systems. Applied Mathematical Modelling 15(10): 534-541.

50. Gelenbe, E. \& Sungur, M. (1994). Random network learning and image compression. In Neural Networks, 1994. IEEE World Congress on Computational Intelligence, Orlando, USA, 1994. IEEE, vol. 6, pp. 3996-3999.

51. Gelenbe, E., Sungur, M., Cramer, C., \& Gelenbe, P. (1996). Traffic and video quality with adaptive neural compression. Multimedia Systems 4(6): 357-369.

52. Gelenbe, E., Tiberio, P., \& Boekhorst, J.C.A. (1973). Page size in demand-paging systems. Acta Informatica 3: 1-23.

53. Gelenbe, E. \& Timotheou, S. (2008). Random neural networks with synchronized interactions. Neural Computation 20(9): 2308-2324.

54. Gelenbe, E. \& Wu, F.-J. (2012). Large scale simulation for human evacuation and rescue. Computers E Mathematics with Applications 64(12): 3869-3880.

55. Gorbil, G., Abdelrahman, O.H., \& Gelenbe, E. (2015). Modeling and analysis of RRC-based signaling storms in $3 \mathrm{~g}$ networks. IEEE Transactions on Emerging Topics in Computing, Special Issue on Emerging Topics in Cyber Security 1: 1-14.

56. Gorbil, G. \& Gelenbe, E. (2011). Opportunistic communications for emergency support systems. Procedia Computer Science 5: 39-47. 